OPEN ACCESS

Edited by:

W. Martin Usrey,

University of California, Davis, USA

Reviewed by:

Elizabeth Quinlan,

University of Maryland College Park,

USA

Marianne Dieterich,

Ludwig-Maximilians-University,

Germany

${ }^{*}$ Correspondence:

Aaron J. Camp

aaron.camp@sydney.edu.au

Received: 28 August 2015 Accepted: 10 November 2015

Published: 02 December 2015

Citation:

Wijesinghe R, Protti DA and Camp AJ

(2015) Vestibular Interactions in

the Thalamus.

Front. Neural Circuits 9:79. doi: 10.3389/fncir.2015.00079

\section{Vestibular Interactions in the Thalamus}

\author{
Rajiv Wijesinghe ${ }^{1}$, Dario A. Protti ${ }^{2}$ and Aaron J. Camp ${ }^{1 *}$ \\ 1 Sensory Systems and Integration Laboratory, Sydney Medical School, Discipline of Biomedical Science, University of \\ Sydney, Sydney, NSW, Australia, ${ }^{2}$ Vision Laboratory, Sydney Medical School, Discipline of Physiology, University of Sydney, \\ Sydney, NSW, Australia
}

It has long been known that the vast majority of all information en route to the cerebral cortex must first pass through the thalamus. The long held view that the thalamus serves as a simple hi fidelity relay station for sensory information to the cortex, however, has over recent years been dispelled. Indeed, multiple projections from the vestibular nuclei to thalamic nuclei (including the ventrobasal nuclei, and the geniculate bodies)regions typically associated with other modalities- have been described. Further, some thalamic neurons have been shown to respond to stimuli presented from across sensory modalities. For example, neurons in the rat anterodorsal and laterodorsal nuclei of the thalamus respond to visual, vestibular, proprioceptive and somatosensory stimuli and integrate this information to compute heading within the environment. Together, these findings imply that the thalamus serves crucial integrative functions, at least in regard to vestibular processing, beyond that imparted by a "simple" relay. In this mini review we outline the vestibular inputs to the thalamus and provide some clinical context for vestibular interactions in the thalamus. We then focus on how vestibular inputs interact with other sensory systems and discuss the multisensory integration properties of the thalamus.

Keywords: vestibular, thalamus, LGN, multisensory integration, vestibular nuclei

\section{INTRODUCTION}

The vestibular system differs from the other primary sensory systems in a number of fundamental ways. Most sensory systems are organized in a linear fashion, where peripheral organ fibers project primarily through a modality-specific thalamic nucleus (for example LGN for the visual system, MGN for the auditory system) and only then onto their respective cortical or subcortical targets. These ordered projections through the thalamus create a sensory map that closely matches that created in the periphery, and this tends to be maintained by downstream thalamocortical projections [for review, see Jones (1985)].

\footnotetext{
Abbreviations: CL, Centrolateral Nucleus; CM, Centromedian Nucleus; IL, Interlaminar Nuclei; IVN, Inferior Vestibular Nucleus; LGN, Lateral Geniculate Nucleus; LVN, Lateral Vestibular Nucleus; LP, Lateral Posterior Nucleus; LD, Lateral Dorsal Nucleus; MGN, Medial Geniculate Nucleus; MVN, Medial Vestibular Nucleus; PNF, Parafascicular Nucleus; SpVN, spinal (descending) vestibular Nucleus; SuVN, Superior Vestibular Nucleus; VA, Ventroanterior Nucleus; VI, Ventral Intermediate Nucleus; VL, Ventrolateral Nucleus; VP, Ventral Posterior Nucleus; VPL, Ventral Posterior Lateral Nucleus; VPM, Ventral Posterior Medial Nucleus.
} 
This organization, along with ordered corticothalamic feedback mechanisms, allows the thalamus to play filtering and modulatory roles in sensory processing (see Sherman, 2007 and references within). Hair cells within the vestibular epithelia are oriented such that they are direction-selective (Lindeman, 1969), however when afferent fibers that relay information from these cells in the semicircular canals, utricle and saccule all converge onto the brainstem vestibular nuclei this signal then becomes more complex. For example, some thalamic neurons receiving vestibular information appear to selectively fire when the organism's head is orientated in a specific direction within the environment, much like a compass (for comprehensive review, see Wiener and Taube, 2005). The brainstem vestibular nuclei project directly to the thalamus, however there is no dedicated vestibular thalamic nucleus that contains a direction or orientation selective map. Further, vestibular thalamic projections are widely distributed to subcortical (Lai et al., 2000), cerebellar (Kotchabhakdi and Walberg, 1978) and cortical (de Waele et al., 2001; Miyamoto et al., 2005) regions that have been shown to be involved in vestibular processing.

Cortical processing of vestibular information is poorly understood compared to other sense modalities such as vision, audition and touch. The perceptual correlate of vestibular activation does not usually correspond to a unimodal sensation, as it occurs with vision and audition. Natural activation of the vestibular system due to head motion and locomotion typically involves activation of visual, vestibular and somatosensory systems and consequently the resulting perceptual correlate are sensations that involve visual, vestibular and somatosensory characteristics. Vestibular-responsive neurons were initially described in somatosensory cortex in areas 3a (Grusser et al., 1990) and area 2v (Buttner and Buettner, 1978). More recently, head direction neurons responsive to vestibular stimulation have also been identified in regions within the classical Papez circuit. Whether signals from different modalities reach these regions independently or they converge at earlier stages is not completely understood yet. There is, however, evidence consistent with integration at the level of the thalamus.

These fundamental observations highlight that the vestibular system has a unique structure amongst the sensory systems, and raises some important questions. In particular, what role does the thalamus play in vestibular processing? In this mini-review we will explore the current understanding of vestibular thalamic function. In the first section, we summarize anatomical studies of vestibulothalamic projections, and suggest that they may be organized into modalityspecific processing streams that permit multisensory integration. In the second section, we analyze physiological studies exploring how vestibular thalamic neurons respond to vestibular information, and clinical observations that give us insights into vestibular processing within the thalamus. In the final section, we make hypotheses about the mechanisms of multisensory integration using the vestibular system as an example, and suggest that the thalamus is well placed to represent a unique subcortical locus of multisensory integration.

\section{VESTIBULOTHALAMIC PROJECTIONS}

Peripheral vestibular stimulation has been shown to cause strong activation within the thalamus (Deecke et al., 1974; Buttner and Henn, 1976; Blum et al., 1979). Tracing studies performed in rat (Shiroyama et al., 1999), cat (Kotchabhakdi et al., 1980), and monkey (Meng et al., 2007), as well as radiological investigations in humans (Kirsch et al., 2015) have demonstrated multiple projections from vestibular nuclei to the thalamus. The primary thalamic targets are the ventrobasal, ventrolateral and intralaminar nuclei and the geniculate bodies (for a recent review, see Lopez and Blanke, 2011). Many of these nuclei are not specific to the vestibular system, and therefore receive inputs from a number of different peripheral sensory and cortical regions. There is some evidence to suggest that these vestibulothalamic circuits may form discrete, functionally specialized pathways that integrate vestibular with other modality-specific signals within the thalamus (Lopez and Blanke, 2011).

Inter alia, the ventrobasal nuclei (ventral posterolateral, VPL; ventral posteromedial, VPM; and ventral intermediate, VI) receive dominant vestibular inputs from bilateral superior vestibular nucleus $(\mathrm{SuVN})$, and contralateral medial vestibular nucleus (MVN) via the medial longitudinal fasciculus (MLF; Nagata, 1986; Matesz et al., 2002). VPM neurons have been shown to respond directly to vestibular nerve stimulation, as well as simulated translational and rotational movements (Marlinski and Mccrea, 2008). These nuclei in turn project to well known vestibular cortical areas such as the anterior suprasylvian cortex (Deecke et al., 1979), posterior cruciate region and the intraparietal sulcus (for review, see Brandt and Dieterich, 1999). The ventral posterior nuclei (VP or VPP) in particular is the source of the major projection to a well studied vestibular cortical region in the macaque monkey, the PIVC (Akbarian et al., 1992). However, it has been shown that some posterior thalamic nuclei such as the VP are also activated during deep somatic and cutaneous stimulation (Deecke et al., 1977). In addition, the VPI projects directly to primary somatosensory cortex (Deecke et al., 1974), as well as vestibular regions of the secondary association somatosensory cortex $(3 \mathrm{aV})$ and posterior parietal cortex (Matsuzaki et al., 2004). These observations show that the thalamus contains the neural circuits necessary to mediate the integration of vestibular and somatosensory signals.

The ventrolateral nuclei (ventral anterior, VA; ventral lateral, VL) receive inputs primarily from LVN, MVN and SuVN via the medial longitudinal fasciculus (MLF; Maciewicz et al., 1982; Nakano et al., 1985; Nagata, 1986). Interestingly, the VL also receives inputs from vestibular cerebellar nuclei such as the fastigial and dentate nuclei via the superior cerebellar peduncle (Sawyer et al., 1994a,b), while the VA receives strong inputs from the basal ganglia (Percheron et al., 1996). The VA-VL complex projects to primary motor and premotor cortices (Brodmann 4 and 6), suggesting that this circuit may represent a major vestibulomotor pathway. The intralaminar nuclei (CL or PFN in rat, $\mathrm{CM}$ ) receive inputs primarily from ipsilateral MVN, SuVN and spinal vestibular nucleus (SpVN; 
Magnin and Kennedy, 1979; Matsuo et al., 1994). Specifically, the PFN has been shown to be a crucial synapse for a vestibulo-thalamo-striatal pathway proposed to be involved in controlling body and limb movements (for review, see Stiles and Smith, 2015). Further, the geniculate bodies (MGN, LGN, SGN) receive inputs from DVN, SuVN and MVN (Kotchabhakdi et al., 1980; Mergner et al., 1981). Despite their primary role in auditory and visual signalling (MGN and LGN respectively), these centers have also been shown to respond to vestibular signals (Liedgren and Schwarz, 1976; RoucouxHanus and Boisacq-Schepens, 1977), suggesting that these thalamic nuclei may participate in subcortical multi-sensory integration. Figure 1 illustrates some of the major pathways connecting the central vestibular nuclei and the thalamus. Note that most of the inputs terminate in the lateral nuclei of the thalamus.

\section{THALAMIC VESTIBULAR PROCESSING}

The vestibular nuclei project heavily to the spinal cord and cerebellum, and these pathways mediate important reflexes that maintain postural control and motor learning (Granit and Pompeiano, 1979; Pompeiano and Allum, 1988). As is evident from the discussion above, there are multiple, divergent vestibular projections that course through the thalamus. However there is limited information about what role the thalamus plays (in concert with the vestibular system) in postural control, and only recently has work begun to delineate its fundamental vestibular processing characteristics.

Clinical observations provide some useful insights into the thalamic contribution to vestibular processing. Postural disorders are increasingly well recognized in patients recovering from strokes, some of which cannot solely be attributed to the unilateral loss of muscular control. For example, Pusher syndrome is an active righting movement observed following strokes where the patient "push[es] actively with non-paretic extremities to the side contralateral to the brain lesion" (Karnath et al., 2005). The lesion induces an abnormality in the perception of the body's alignment in relation to gravity, and has been described in patients with circumscribed posterior thalamic lesions as well as other adjacent brain structures (Karnath et al., 2005). Specifically, patients with pusher syndrome perceive their body to be positioned vertically despite approximately 18 degrees

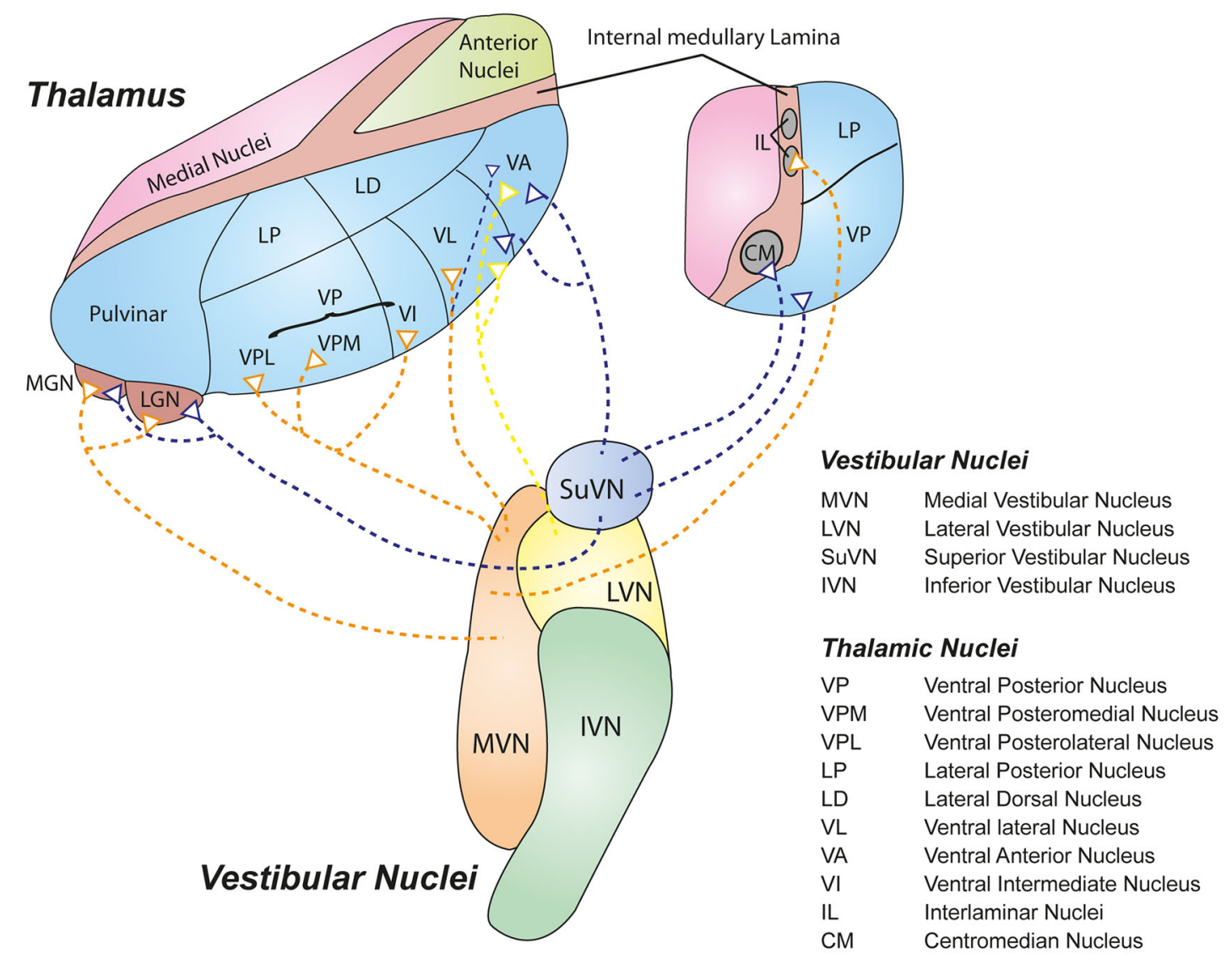

FIGURE 1 | Schematic representation of some of the major vestibulothalamic projections. Defined projections from the four main vestibular nuclei to their thalamic targets are shown. Note that the vast majority of vestibulothalamic projections originate in the medial vestibular nucleus (MVN; orange) and SuVN (mauve), and terminate in the lateral nuclei of the thalamus (Blue). 
of tilt of the subjective postural vertical in the roll plane (for review see Karnath, 2007). Interestingly the subjective visual vertical appears remarkably undisturbed in pusher syndrome (Karnath et al., 2000). The observation that cortical infarcts are also capable of inducing this syndrome (Johannsen et al., 2006; Karnath et al., 2008) or other perturbations of subjective visual vertical (Baier et al., 2012) suggests that the posterior thalamus is important but not unique in generating this perception of verticality. Another interesting insight comes from studies of patients suffering from vestibular migraine, a central vestibular disorder characterized by an abnormal vestibular percept without peripheral vestibulopathy (for recent review, see Stolte et al., 2015). Functional magnetic resonance imaging (fMRI) studies in these patients during vestibular stimulation demonstrated increased BOLD signal within the thalamus compared to both healthy controls and migraineurs with aura (Russo et al., 2014). These observations demonstrate that the thalamus plays in integral role in the generation of complex vestibular percepts, and that abnormalities in thalamic processing may account for the distortion of these percepts observed in certain clinical situations.
A number of in vivo electrophysiology studies have demonstrated the vestibular evoked responses of thalamic neurons. Meng et al. (2007) recorded the responses of thalamic neurons in the ventral posterior and ventral lateral thalamic nuclei of macaque monkeys. In general, they found that vestibular-responsive thalamic neurons show mixed selectivity for rotations and translations within different planes, however they tend to be more responsive to translations within the yaw plane. Subsequently, a small subset of thalamic neurons was found to be responsive to both vestibular and visual inputs (Meng and Angelaki, 2010). Figure 2 shows the translation direction tuning to both visual and vestibular stimulation for one example multisensory neuron. Similar responses were also observed for rotational stimuli. In addition, experiments by Marlinski and colleagues demonstrated that neurons tended to respond either to ispi- or contralateral rotation, with no neurons displaying bidirectional sensitivity (Marlinski and Mccrea, 2008). Experiments performed in both light and dark environments demonstrate that the vestibular signal in the thalamus is independent of visual signals, however the addition of visual information does

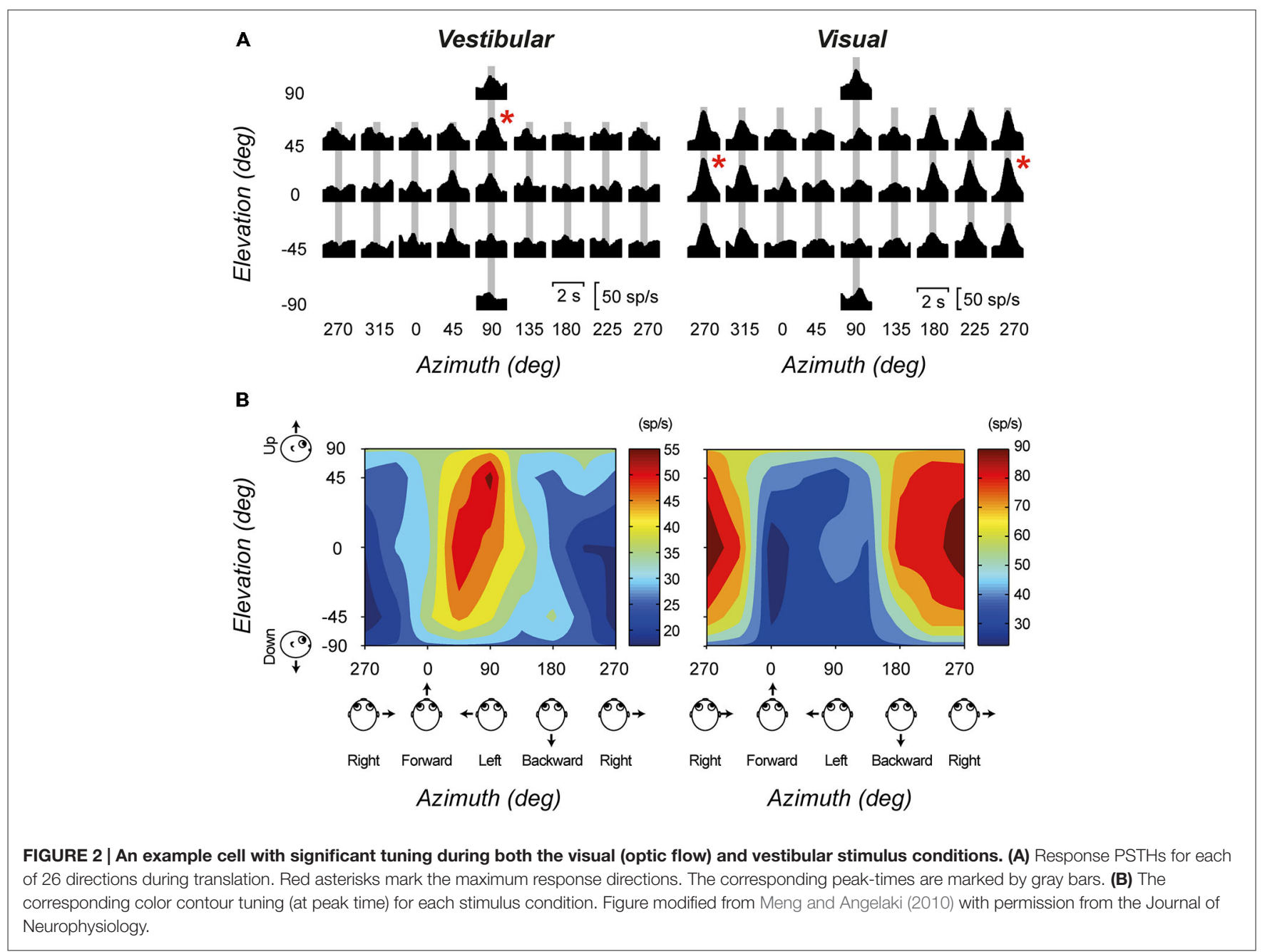


increase the sensitivity of these neurons to incoming inputs. Both groups showed a large range of responsiveness to rotational stimulation, in both amplitude and phase. Their experiments demonstrated a large number of vestibular responsive areas within the thalamus, outside of the classically described vestibulothalamic projection zones. Interestingly, Marlinski and colleagues demonstrated small clusters of vestibular-responsive neurons within the VP nucleus, and postulate that these may be analog to the discrete regions seen within the macaque thalamus that project to specific vestibular-specific cortical regions (Marlinski and Mccrea, 2008).

Few investigators have studied the intrinsic properties of neurons within the thalamus that receive vestibular inputs. One recent study by Kulkarni and colleagues examined the properties of rat anterodorsal thalamic neurons in the presence of synaptic blockade (Kulkarni et al., 2011). They found that single neurons were able to sustain long periods of regular firing over many minutes with minimal adaptation. This response was particularly evident when induced by hyperpolarizing as opposed to depolarizing pulses. Work studying the synaptic profile of anterodorsal thalamic neurons suggests that they are characteristic of those within a first order relay nucleus, with a driver input from the mammillary bodies and modulatory input from cortical feedback (Petrof and Sherman, 2009). It would be interesting to see whether these neurons also display similar output profiles to other first-order relay thalamocortical neurons such as those seen in the lateral geniculate nucleus (Gutierrez et al., 2001; Wijesinghe et al., 2013).

\section{MULTISENSORY INTEGRATION IN THE THALAMUS}

Perceptually, the vestibular system is integral in generating higher order sensory phenomena that require input from other sensory systems. For example, creating the sense of the visual vertical requires input from both visual and vestibular neurons (Vingerhoets et al., 2009); postural stability requires input from proprioceptive neurons and vestibular neurons (Bronstein, 1999). Studies have also unexpectedly suggested a role for the vestibular system in cognitive domains such as memory and learning (Smith and Zheng, 2013) and body representation (Mast et al., 2014). As outlined above, thalamic nuclei receiving vestibular afferents are also heavily involved in the processing of information from other sensory systems. This raises the possibility that the thalamus may serve as an important locus for multisensory integration. Indeed, a recent study shows tantalizing evidence that driver (primary and secondary vestibular) and modulatory (visual) inputs onto cerebellar granule cells have different strength and temporal course. In addition, when these inputs are co-activated they produce an enhanced response with a characteristic first spike latency that allows temporal coding of multisensory inputs (Chabrol et al., 2015). It is tempting to speculate, as the authors do, that similar mechanisms may also be involved in the integration of synaptic inputs of different sensory modalities in other thalamic neurons and HD cells that receive vestibular information.

Studies comparing stroke patients with a degree of somatosensory loss to normal controls have suggested that interactions between vestibular and somatosensory information is dependent on the function of the posterolateral thalamus (Barra et al., 2010). Further, psychophysical studies have demonstrated that multisensory integration is impaired in patients with Parkinson's disease, possibly be due to thalamic dysfunction induced by lack of facilitation by ascending cholinergic systems (Muller et al., 2013). Interestingly, one study reporting on two patients with Parkinson's following deep brain stimulation of the subthalamic nucleus showed changes in the subjective visual vertical (Mike et al., 2009)a feature typically associated with peripheral and/or central vestibular dysfunction- again suggestive of vestibular thalamic interactions.

Experimentally, a model system for multisensory integration is the head-direction (HD) system, which contains neurons that encode head orientation in the horizontal (yaw) plane independently of location within an environment (for review, see Taube, 2007). HD neurons have been found in a number of regions within the classical Papez circuit, such as the anterodorsal thalamus, lateral mammillary nuclei, and the retrosplenial and entorhinal cortices (see Wiener and Taube, 2005 and references within for details). The vestibular system plays an integral role in the generation of the HD signal, as demonstrated by behavioral studies assessing spatial navigation while restricting sensory cues or with specific brain regions deactivated (Yoder and Taube, 2014). A recent in vivo study analyzing the headdirection cell response to both active and passive movements has shown that vestibular input is necessary and sufficient to generate the HD signal (Shinder and Taube, 2011), highlighting the central role of the vestibular system in this localization pathway.

Thalamic HD neurons have been the focus of recent investigations aiming to elucidate the specific mechanisms behind the HD signal. Using rats on a moving treadmill, Enkhjargal et al. (2014) studied the effect of more complex movements involving multiple frames of reference with conflicting sensory inputs. They found that thalamic HD neurons displayed complex spatial firing patterns that depended on the combinations of facing direction and movement direction; that is, their activity depends on perceived directional heading, optic flow, vestibular and proprioceptive information (Enkhjargal et al., 2014). Interestingly, the directionality seen with the head-direction signal may be dependent on the intrinsic properties of anterodorsal thalamic neurons, specifically their propensity for irregular firing (Tsanov et al., 2014). The finding that the HD signal is also present when motionless (Shinder and Taube, 2014) may suggest that intrinsic mechanisms are capable of maintaining the HD signal once it has been generated. Recent investigations analyzing ensembles of $\mathrm{HD}$-cells within the anterodorsal nucleus and post-subiculum found coherent activations during awake and sleep network states, suggesting that the $\mathrm{HD}$ representation may be generated internally within 
thalamic circuits and modulated by sensory inputs (Peyrache et al., 2015). Another exciting new discovery has shown that parahippocampal grid cell activity may be dependent upon the thalamic head direction signal (Winter et al., 2015), highlighting a central role of the thalamus as a major locus for multisensory integration in the generation of complex perceptual phenomena, particularly in regard to the vestibular contributions to navigation.

\section{SUMMARY}

Our understanding of the vestibular interactions within the thalamus remains nascent, however is growing quite quickly. Clinical and experimental observations are piecing together a clearer picture of how vestibular signals are processed and combined with other sensory signals to form complex representations of the environment and our actions within it. Despite this, the underlying cellular mechanisms that mediate these processes remain unclear. What are the characteristics of individual thalamic neurons in these multisensory pathways? What are the intrinsic properties of these neurons that allow

\section{REFERENCES}

Akbarian, S., Grusser, O. J., and Guldin, W. O. (1992). Thalamic connections of the vestibular cortical fields in the squirrel monkey (Saimiri sciureus). J. Comp. Neurol. 326, 423-441.

Baier, B., Suchan, J., Karnath, H. O., and Dieterich, M. (2012). Neural correlates of disturbed perception of verticality. Neurology 78, 728-735. doi: 10.1212/wnl. 0b013e318248e 544

Barra, J., Marquer, A., Joassin, R., Reymond, C., Metge, L., Chauvineau, V., et al. (2010). Humans use internal models to construct and update a sense of verticality. Brain 133, 3552-3563. doi: 10.1093/brain/awq311

Blum, P. S., Day, M. J., Carpenter, M. B., and Gilman, S. (1979). Thalamic components of the ascending vestibular system. Exp. Neurol. 64, 587-603. doi: 10.1016/0014-4886(79)90234-6

Brandt, T., and Dieterich, M. (1999). The vestibular cortex. Its locations, functions and disorders. Ann. N Y Acad. Sci. 871, 293-312. doi: 10.1007/978-1-44710527-5_13

Bronstein, A. M. (1999). The interaction of otolith and proprioceptive information in the perception of verticality. The effects of labyrinthine and CNS disease. Ann. N Y Acad. Sci. 871, 324-333. doi: 10.1111/j.1749-6632.1999. tb09195.x

Buttner, U., and Buettner, U. W. (1978). Parietal cortex (2v) neuronal activity in the alert monkey during natural vestibular and optokinetic stimulation. Brain Res. 153, 392-397. doi: 10.1016/0006-8993(78)90421-3

Buttner, U., and Henn, V. (1976). Thalamic unit activity in the alert monkey during natural vestibular stimulation. Brain Res. 103, 127-132. doi: 10. 1016/0006-8993(76)90692-2

Chabrol, F. P., Arenz, A., Wiechert, M. T., Margrie, T. W., and Digregorio, D. A. (2015). Synaptic diversity enables temporal coding of coincident multisensory inputs in single neurons. Nat. Neurosci. 18, 718-727. doi: 10.1038/nn.3974

de Waele, C., Baudonniere, P. M., Lepecq, J. C., Tran Ba Huy, P., and Vidal, P. P. (2001). Vestibular projections in the human cortex. Exp. Brain Res. 141, 541-551. doi: 10.1007/s00221-001-0894-7

Deecke, L., Mergner, T., and Becker, W. (1979). Neuronal responses to natural vestibular stimuli in the cat's anterior suprasylvian gyrus. Adv. Otorhinolaryngol. 25, 74-81. doi: 10.1016/0006-8993(79)90052-0

Deecke, L., Schwarz, D. W., and Fredrickson, J. M. (1974). Nucleus ventroposterior inferior (VPI) as the ventibular thalamic relay in the rhesus monkey. I. Field potential investigation. Exp. Brain Res. 20, 88-100. doi: 10.1007/bf002 39019 this filtering and integration to take place? What synaptic and intrinsic properties mediate the processing of disparate and conflicting signals from different sensory systems? What is clear, however, is that the thalamus is a point of convergence for signals from essentially all sensory modalities, and that groups of, and even individual neurons within the Thalamus, have the capacity to compute a variety of output functions depending on the signals received. This feature alone is sufficient to clearly define the Thalamus as "beyond a simple relay".

\section{FUNDING}

The authors would also like to acknowledge The Support of the Garnett Passe and Rodney Williams Memorial Foundation.

\section{ACKNOWLEDGMENTS}

The authors would like to thank Ms Victoria Tung and Ms Miranda Mathews for comments on previous versions of this manuscript.

Deecke, L., Schwarz, D. W., and Fredrickson, J. M. (1977). Vestibular responses in the rhesus monkey ventroposterior thalamus. II. Vestibulo-proprioceptive convergence at thalamic neurons. Exp. Brain Res. 30, 219-232. doi: 10. $1007 /$ bf00237252

Enkhjargal, N., Matsumoto, J., Chinzorig, C., Berthoz, A., Ono, T., and Nishijo, H. (2014). Rat thalamic neurons encode complex combinations of heading and movement directions and the trajectory route during translocation with sensory conflict. Front. Behav. Neurosci. 8:242. doi: 10.3389/fnbeh.2014.00242

Granit, R., and Pompeiano, O. (1979). Reflex Control of Posture and Movement Holland: Elsevier.

Grusser, O. J., Pause, M., and Schreiter, U. (1990). Localization and responses of neurons in the parieto-insular vestibular cortex of awake monkeys (macacafascicularis). J. Physiol. 430, 537-557.

Gutierrez, C., Cox, C. L., Rinzel, J., and Sherman, S. M. (2001). Dynamics of lowthreshold spike activation in relay neurons of the cat lateral geniculate nucleus. J. Neurosci. 21, 1022-1032.

Johannsen, L., Broetz, D., Naegele, T., and Karnath, H. O. (2006). "Pusher syndrome" following cortical lesions that spare the thalamus. J. Neurol 253, 455-463. doi: 10.1007/s00415-005-0025-7

Jones, E. G. (1985). The Thalamus. New York and London: Plenum Press.

Karnath, H. O. (2007). Pusher syndrome-a frequent but little-known disturbance of body orientation perception. J. Neurol. 254, 415-424. doi: 10.1007/s00415006-0341-6

Karnath, H. O., Ferber, S., and Dichgans, J. (2000). The origin of contraversive pushing: evidence for a second graviceptive system in humans. Neurology 55, 1298-1304. doi: 10.1212/wnl.55.9.1298

Karnath, H. O., Johannsen, L., Broetz, D., and Kuker, W. (2005). Posterior thalamic hemorrhage induces "pusher syndrome". Neurology 64, 1014-1019. doi: $10.1212 /$ wnl.65.10.1682

Karnath, H. O., Suchan, J., and Johannsen, L. (2008). Pusher syndrome after ACA territory infarction. Eur. J. Neurol. 15, e84-e85. doi: 10.1111/j.1468-1331.2008. 02187.x

Kirsch, V., Keeser, D., Hergenroeder, T., Erat, O., Ertl-Wagner, B., Brandt, T., et al. (2015). Structural and functional connectivity mapping of the vestibular circuitry from human brainstem to cortex. Brain Struct. Funct. doi: 10. 1007/s00429-014-0971-x [Epub ahead of print].

Kotchabhakdi, N., Rinvik, E., Walberg, F., and Yingchareon, K. (1980). The vestibulothalamic projections in the cat studied by retrograde axonal transport of horseradish peroxidase. Exp. Brain Res. 40, 405-418. doi: 10. 1007/bf00236149 
Kotchabhakdi, N., and Walberg, F. (1978). Cerebellar afferent projections from the vestibular nuclei in the cat: an experimental study with the method of retrograde axonal transport of horseradish peroxidase. Exp. Brain Res. 31, 591-604. doi: 10.1007/bf00239814

Kulkarni, M., Zhang, K., and Kirkwood, A. (2011). Single-cell persistent activity in anterodorsal thalamus. Neurosci. Lett. 498, 179-184. doi: 10.1016/j.neulet.2011. 02.051

Lai, H., Tsumori, T., Shiroyama, T., Yokota, S., Nakano, K., and Yasui, Y. (2000). Morphological evidence for a vestibulo-thalamo-striatal pathway via the parafascicular nucleus in the rat. Brain Res. 872, 208-214. doi: 10. 1016/s0006-8993(00)02457-4

Liedgren, S. R., and Schwarz, D. W. (1976). Vestibular evoked potentials in thalamus and basal ganglia of the squirrel monkey (Saimiri sciureus). Acta. Otolaryngol. 81, 73-82.

Lindeman, H. H. (1969). Studies on the morphology of the sensory regions of the vestibular apparatus with 45 figures. Ergeb. Anat. Entwicklungsgesch. 42, 1-113. doi: $10.1007 / 978-3-662-24992-5$

Lopez, C., and Blanke, O. (2011). The thalamocortical vestibular system in animals and humans. Brain Res. Rev. 67, 119-146. doi: 10.1016/j.brainresrev.2010. 12.002

Maciewicz, R., Phipps, B. S., Bry, J., and Highstein, S. M. (1982). The vestibulothalamic pathway: contribution of the ascending tract of Deiters. Brain Res. 252, 1-11. doi: 10.1016/0006-8993(82)9 0973-8

Magnin, M., and Kennedy, H. (1979). Anatomical evidence of a third ascending vestibular pathway involving the ventral lateral geniculate nucleus and the intralaminar nuclei of the cat. Brain Res. 171, 523-529. doi: 10.1016/00068993(79)91056-4

Marlinski, V., and Mccrea, R. A. (2008). Activity of ventroposterior thalamus neurons during rotation and translation in the horizontal plane in the alert squirrel monkey. J. Neurophysiol. 99, 2533-2545. doi: 10.1152/jn.00761. 2007

Mast, F. W., Preuss, N., Hartmann, M., and Grabherr, L. (2014). Spatial cognition, body representation and affective processes: the role of vestibular information beyond ocular reflexes and control of posture. Front. Integr. Neurosci. 8:44. doi: $10.3389 /$ fnint.2014.00044

Matesz, C., Bacskai, T., Nagy, E., Halasi, G., and Kulik, A. (2002). Efferent connections of the vestibular nuclei in the rat: a neuromorphological study using PHA-L. Brain Res. Bull. 57, 313-315. doi: 10.1016/s0361-9230(01) 00726-2

Matsuo, S., Hosogai, M., and Nakao, S. (1994). Ascending projections of posterior canal-activated excitatory and inhibitory secondary vestibular neurons to the mesodiencephalon in cats. Exp. Brain Res. 100, 7-17. doi: 10.1007/bf002 27274

Matsuzaki, R., Kyuhou, S., Matsuura-Nakao, K., and Gemba, H. (2004). Thalamo-cortical projections to the posterior parietal cortex in the monkey. Neurosci. Lett. 355, 113-116. doi: 10.1016/j.neulet.2003. 10.066

Meng, H., and Angelaki, D. E. (2010). Responses of ventral posterior thalamus neurons to three-dimensional vestibular and optic flow stimulation. J. Neurophysiol. 103, 817-826. doi: 10.1152/jn.00729.2009

Meng, H., May, P. J., Dickman, J. D., and Angelaki, D. E. (2007). Vestibular signals in primate thalamus: properties and origins. J. Neurosci. 27, 13590-13602. doi: 10.1523/jneurosci.3931-07.2007

Mergner, T., Deecke, L., and Wagner, H. J. (1981). Vestibulo-thalamic projection to the anterior suprasylvian cortex of the cat. Exp. Brain Res. 44, 455-458. doi: $10.1007 /$ bf00238841

Mike, A., Balas, I., Varga, D., Janszky, J., Nagy, F., and Kovacs, N. (2009). Subjective visual vertical may be altered by bilateral subthalamic deep brain stimulation. Mov. Disord. 24, 1556-1557. doi: 10.1002/mds. 22605

Miyamoto, T., Fukushima, K., Takada, T., de Waele, C., and Vidal, P. P. (2005). Saccular projections in the human cerebral cortex. Ann. N Y Acad. Sci. 1039, 124-131. doi: 10.1196/annals.1325.012

Muller, M. L., Albin, R. L., Kotagal, V., Koeppe, R. A., Scott, P. J., Frey, K. A., et al. (2013). Thalamic cholinergic innervation and postural sensory integration function in Parkinson's disease. Brain 136, 3282-3289. doi: 10. 1093/brain/awt247
Nagata, S. (1986). The vestibulothalamic connections in the rat: a morphological analysis using wheat germ agglutinin-horseradish peroxidase. Brain Res. 376, $57-70$.

Nakano, K., Kohno, M., Hasegawa, Y., and Tokushige, A. (1985). Cortical and brain stem afferents to the ventral thalamic nuclei of the cat demonstrated by retrograde axonal transport of horseradish peroxidase. J. Comp. Neurol. 231, 102-120. doi: 10.1002/cne.902310109

Percheron, G., Francois, C., Talbi, B., Yelnik, J., and Fenelon, G. (1996). The primate motor thalamus. Brain Res. Brain Res. Rev. 22, 93-181. doi: 10. 1016/s0165-0173(96)00003-3

Petrof, I., and Sherman, S. M. (2009). Synaptic properties of the mammillary and cortical afferents to the anterodorsal thalamic nucleus in the mouse. J. Neurosci. 29, 7815-7819. doi: 10.1523/jneurosci.1564-09.2009

Peyrache, A., Lacroix, M. M., Petersen, P. C., and Buzsaki, G. (2015). Internally organized mechanisms of the head direction sense. Nat. Neurosci. 18, 569-575. doi: $10.1038 / \mathrm{nn} .3968$

Pompeiano, O., and Allum, J. H. J. (1988). Vestibulospinal Control of Posture and Locomotion Holland: Elsevier.

Roucoux-Hanus, M., and Boisacq-Schepens, N. (1977). Ascending vestibular projections: further results at cortical and thalamic levels in the cat. Exp. Brain Res. 29, 283-292. doi: 10.1007/bf00237048

Russo, A., Marcelli, V., Esposito, F., Corvino, V., Marcuccio, L., Giannone, A., et al. (2014). Abnormal thalamic function in patients with vestibular migraine. Neurology 82, 2120-2126. doi: 10.1212/wnl.0000000000 000496

Sawyer, S. F., Tepper, J. M., and Groves, P. M. (1994a). Cerebellar-responsive neurons in the thalamic ventroanterior-ventrolateral complex of rats: light and electron microscopy. Neuroscience 63, 725-745. doi: 10.1016/03064522(94)90518-5

Sawyer, S. F., Young, S. J., Groves, P. M., and Tepper, J. M. (1994b). Cerebellarresponsive neurons in the thalamic ventroanterior-ventrolateral complex of rats: in vivo electrophysiology. Neuroscience 63, 711-724. doi: 10.1016/03064522(94) $90517-7$

Sherman, S. M. (2007). The thalamus is more than just a relay. Curr. Opin. Neurobiol. 17, 417-422. doi: 10.1016/j.conb.2007. 07.003

Shinder, M. E., and Taube, J. S. (2011). Active and passive movement are encoded equally by head direction cells in the anterodorsal thalamus. J. Neurophysiol. 106, 788-800. doi: 10.1152/jn.01098.2010

Shinder, M. E., and Taube, J. S. (2014). Self-motion improves head direction cell tuning. J. Neurophysiol. 111, 2479-2492. doi: 10.1152/jn.00512. 2013

Shiroyama, T., Kayahara, T., Yasui, Y., Nomura, J., and Nakano, K. (1999). Projections of the vestibular nuclei to the thalamus in the rat: a Phaseolus vulgaris leucoagglutinin study. J. Comp. Neurol. 407, 318-332. doi: 10. 1002/(sici)1096-9861(19990510)407:3318::aid-cne23.3.co;2-8

Smith, P. F., and Zheng, Y. (2013). From ear to uncertainty: vestibular contributions to cognitive function. Front. Integr. Neurosci. 7:84. doi: 10 . 3389/fnint.2013.00084

Stiles, L., and Smith, P. F. (2015). The vestibular-basal ganglia connection: balancing motor control. Brain Res. 1597, 180-188. doi: 10.1016/j.brainres. 2014.11.063

Stolte, B., Holle, D., Naegel, S., Diener, H. C., and Obermann, M. (2015). Vestibular migraine. Cephalalgia 35, 262-270. doi: 10.1177/03331024145 35113

Taube, J. S. (2007). The head direction signal: origins and sensory-motor integration. Annu. Rev. Neurosci. 30, 181-207. doi: 10.1146/annurev.neuro.29. 051605.112854

Tsanov, M., Chah, E., Noor, M. S., Egan, C., Reilly, R. B., Aggleton, J. P., et al. (2014). The irregular firing properties of thalamic head direction cells mediate turn-specific modulation of the directional tuning curve. J. Neurophysiol. 112, 2316-2331. doi: 10.1152/jn.00583.2013

Vingerhoets, R. A., de Vrijer, M., Van Gisbergen, J. A., and Medendorp, W. P. (2009). Fusion of visual and vestibular tilt cues in the perception of visual vertical. J. Neurophysiol. 101, 1321-1333. doi: 10.1152/jn.90725. 2008

Wiener, S. I., and Taube, J. S. (2005). Head Direction Cells and the Neural Mechanisms of Spatial Orientation. Cambridge, MA: MIT Press. 
Wijesinghe, R., Solomon, S. G., and Camp, A. J. (2013). Noise normalizes firing output of mouse lateral geniculate nucleus neurons. PLoS One. 8:e57961. doi: 10.1371/journal.pone.0057961

Winter, S. S., Clark, B. J., and Taube, J. S. (2015). Spatial navigation. Disruption of the head direction cell network impairs the parahippocampal grid cell signal. Science 347, 870-874. doi: 10.1126/science. 1259591

Yoder, R. M., and Taube, J. S. (2014). The vestibular contribution to the head direction signal and navigation. Front. Integr. Neurosci. 8:32. doi: 10.3389/fnint. 2014.00032
Conflict of Interest Statement: The authors declare that the research was conducted in the absence of any commercial or financial relationships that could be construed as a potential conflict of interest.

Copyright (c) 2015 Wijesinghe, Protti and Camp. This is an open-access article distributed under the terms of the Creative Commons Attribution License (CC BY). The use, distribution and reproduction in other forums is permitted, provided the original author(s) or licensor are credited and that the original publication in this journal is cited, in accordance with accepted academic practice. No use, distribution or reproduction is permitted which does not comply with these terms. 